\title{
HIGH-ENERGY OBSERVATIONS OF PSR B1259-63/LS 2883 THROUGH THE 2014 PERIASTRON PASSAGE: CONNECTING X-RAYS TO THE GeV FLARE
}

\author{
P. H. T. TAM ${ }^{1,2}$, K. L. Li ${ }^{1}$, J. TAKATA ${ }^{2}$, A. T. OKAZAKI ${ }^{3}$, C. Y. HuI ${ }^{4}$, AND A. K. H. Kong ${ }^{1}$ \\ ${ }^{1}$ Institute of Astronomy and Department of Physics, National Tsing Hua University, Hsinchu, Taiwan; phtam@phys.nthu.edu.tw \\ ${ }^{2}$ Department of Physics, University of Hong Kong, Pokfulam Road, Hong Kong \\ ${ }^{3}$ Faculty of Engineering, Hokkai-Gakuen University, Toyohira-ku, Sapporo 062-8605, Japan \\ ${ }^{4}$ Department of Astronomy and Space Science, Chungnam National University, Daejeon, Korea \\ Received 2014 October 7; accepted 2014 December 5; published 2014 December 19
}

\begin{abstract}
The binary system PSR B1259-63/LS 2883 is well sampled in radio, X-rays, and TeV $\gamma$-rays, and shows orbitalphase-dependent variability in these frequencies. The first detection of $\mathrm{GeV} \gamma$-rays from the system was made around the 2010 periastron passage. In this Letter, we present an analysis of X-ray and $\gamma$-ray data obtained by the Swift/XRT, NuSTAR/FPM, and Fermi/LAT, through the recent periastron passage which occurred on 2014 May 4. While PSR B1259-63/LS 2883 was not detected by the Large Area Telescope before and during this passage, we show that the $\mathrm{GeV}$ flares occurred at a similar orbital phase as in early 2011, thus establishing the repetitive nature of the post-periastron $\mathrm{GeV}$ flares. Multiple flares each lasting for a few days have been observed and short-term variability is seen as well. We also found $\mathrm{X}$-ray flux variation contemporaneous with the $\mathrm{GeV}$ flare for the first time. Strong evidence of the keV-to-GeV connection came from the broadband high-energy spectra, which we interpret as synchrotron radiation from the shocked pulsar wind.
\end{abstract}

Key words: gamma rays: stars - pulsars: individual (PSR B1259-63) - X-rays: binaries

\section{INTRODUCTION}

PSR B1259-63/LS 2883 is a unique binary system in our Galaxy located at $d=2.3 \pm 0.4 \mathrm{kpc}$ away (Negueruela et al. 2011). It comprises the young radio pulsar, PSR B1259-63, with a period $47.8 \mathrm{~ms}$ and the fast-rotating massive star LS 2883 (Johnston et al. 1994). Moving in an eccentric $(e \sim 0.87)$ orbit, the pulsar passes the periastron every 1236.7 days (or $3.4 \mathrm{yr}$; Shannon et al. 2014). LS 2883 hosts a stellar disk that is inclined with respect to the orbital plane, such that the pulsar crosses the disk plane just before and just after each periastron passage (Wex et al. 1998). The pulsar generates a powerful pulsar wind (PW) that interacts with the stellar wind/disk, emitting broadband non-thermal, unpulsed radiation, in radio (e.g., Johnston et al. 2005), X-rays (e.g., Chernyakova et al. 2009), and $\mathrm{TeV} \gamma$-rays (Aharonian et al. 2005, 2009). Because of the highly eccentric orbit, the broadband radiation varies over the $3.4 \mathrm{yr}$ orbit, and peaks around the periastron passages (e.g., see Chernyakova et al. 2006, 2009).

Radio imaging observations have revealed variable spatially extended emission whose size is larger than the binary orbit (Moldón et al. 2011). Chandra observations near apastron also show extended X-ray emission (Pavlov et al. 2011).

Over the last periastron passage which occurred 2010 December, the most surprising behavior came from the $\mathrm{GeV}$ band. It was the first Fermi Large Area Telescope (LAT) observations of the system through a periastron passage. Before and during the passage, the LAT detected a weak emission above $100 \mathrm{MeV}$, before, quite surprisingly, a GeV flare occurred $\sim 30$ days after the passage (Kong et al. 2011a), with a flux about 10 times the pre-periastron value. The flares continued until about three months after the periastron passage (Tam et al. 2011; Abdo et al. 2011).

H.E.S.S. observations of the binary were performed just before and during part of the $\mathrm{GeV}$ flaring period in 2011 January. No significant brightening above $1 \mathrm{TeV}$ was seen, and the flare coefficient, $\kappa_{\mathrm{TeV}}$, i.e., the $>1 \mathrm{TeV}$ flux ratio during the flare and before the flare, is constrained to be $\kappa_{\mathrm{TeV}}<3.5$ at the $99.7 \%$ confidence level (H.E.S.S. Collaboration 2013). No X-ray flare was seen during the $\mathrm{GeV}$ flare as well (e.g., Chernyakova et al. 2014).

The radiation mechanisms of the broadband radiation from PSR B1259-63/LS 2883, as well as the GeV flare seen in early 2011, are unclear. Electrons accelerated in the shock between the PW and stellar wind can produce synchrotron radiation and/or upscatter stellar photons from LS 2883 to produce inverse-Compton (IC) radiation (Tavani \& Arons 1997; Kirk et al. 1999; Dubus 2006; Bogovalov et al. 2008; Khangulyan et al. 2011; Kong et al. 2011b; Mochol \& Kirk 2013; Takata \& Taam 2009; Takata et al. 2012). The unshocked PW particles may also generate $\gamma$-rays (Khangulyan et al. 2012). The interaction between the stellar disk and the pulsar (Chernyakova et al. 2014), as well as Doppler boosting (Dubus et al. 2010; Kong et al. 2012) may also play a role. The extended radio emission around periastron may be generated by particles that have traveled outside the binary system. High-energy observations during the 2014 periastron passage may help us to distinguish competing scenarios.

In this Letter, Fermi/LAT, NuSTAR/FPM, and Swift/XRT analysis results of the binary PSR B1259-63/LS 2883 over the periastron passage which occurred on 2014 May 4 (or, $t_{\mathrm{p}}=$ MJD 56781.4195) are presented.

\section{GAMMA-RAY OBSERVATIONS AND RESULTS}

\subsection{Observations and Data Analysis}

The $\gamma$-ray data ${ }^{5}$ used in this work were obtained using the Fermi/LAT (Atwood et al. 2009) between 2008 August 4 and 2014 August 2. In response to target-of-opportunity requests, observations targeted at PSR B1259-63/LS 2883 were commenced from 2014 May 31 to June 26, such that the exposure toward PSR B1259-63 was increased during the above period. We used the Fermi Science Tools v9r32p5 package to reduce and analyze the data. Reprocessed Pass 7

\footnotetext{
5 Provided by the FSSC at http://fermi.gsfc.nasa.gov/ssc/.
} 


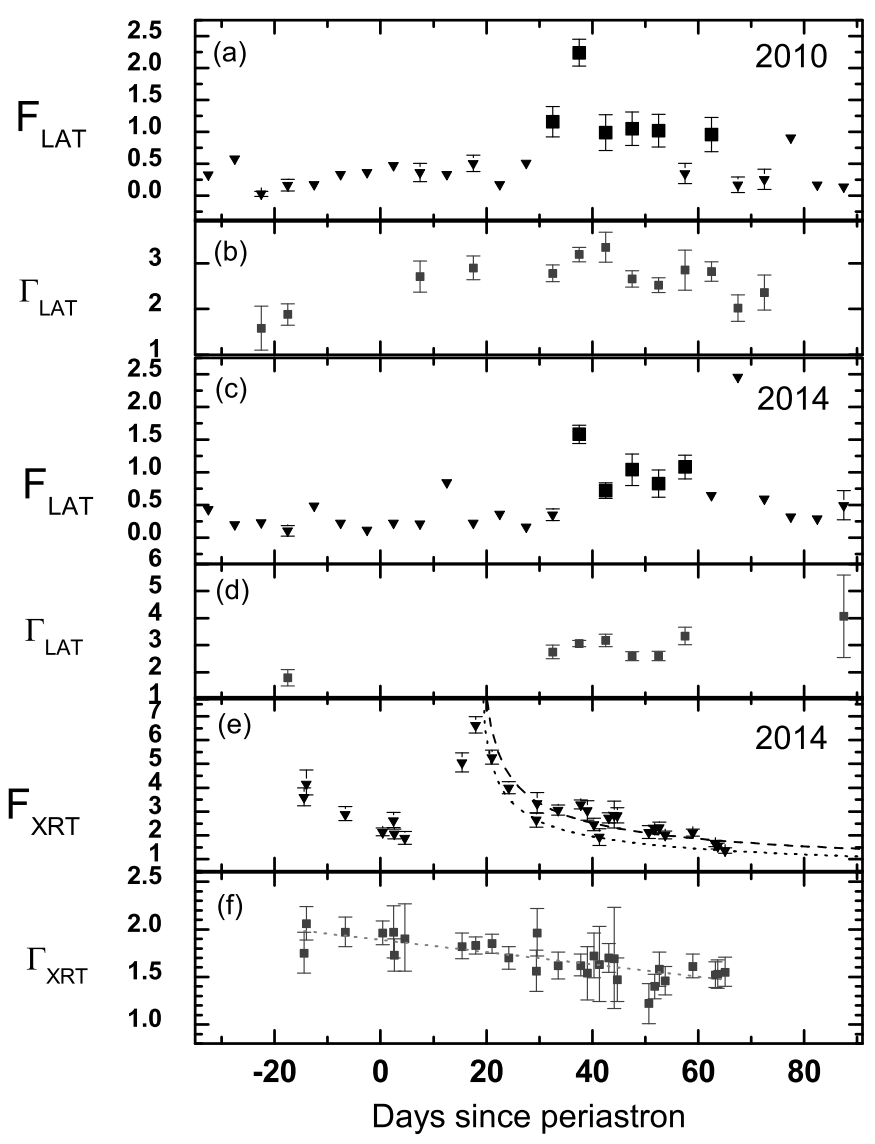

Figure 1. High-energy light curves of PSR B1259-63/LS 2883 over its recent periastron passages. (a) and (c): Fermi/LAT 0.1-300 GeV light curve over the 2010 and 2014 periastron passage, respectively. Flux is given in $10^{-6}$ photons $\mathrm{cm}^{-2} \mathrm{~s}^{-1}$. Five-day time bins are used. Bins with TS $>25$ (significant) are indicated by squares. Bins with $5<\mathrm{TS}<25$ (marginally significant) are indicated by triangles. For bins with TS $<5,95 \%$ confidencelevel upper limits were calculated assuming $\Gamma=3$; (b) and (d): $\gamma$-ray photon index evolution over the 2010 and 2014 periastron passage, respectively; (e) Swift/XRT 0.3-10 keV light curve in 2014. Flux is given in $10^{-11} \mathrm{erg} \mathrm{cm}^{-2} \mathrm{~s}^{-1}$. The best-fit baseline model (dotted) and the declining tend (dashed) are fitted using data after June 2. (f) X-ray photon index evolution in 2014. The dashed line shows the linear fit $\Gamma_{\mathrm{XRT}}=(1.89 \pm 0.04)-(0.006 \pm 0.001)\left[t(d)-t_{\mathrm{p}}\right]$.

data classified as "source" events were used. To reduce the contamination from Earth albedo $\gamma$-rays, we excluded events with zenith angles greater than $100^{\circ}$. The instrument response functions "P7REP_SOURCE_V15" were used.

We first carried out a binned maximum-likelihood analysis (gtlike) of a rectangular region of $21^{\circ} \times 21^{\circ}$ centered on the position of PSR B1259-63/LS 2883, using 6 yr data. We subtracted the background contribution by including the Galactic diffuse model (gll_iem_v05.fit) and the isotropic background (iso_iem_v05.txt), as well as the second Fermi/LAT catalog (2FGL; Nolan et al. 2012) sources within $25^{\circ}$ away from PSR B1259-63. The $\gamma$-ray source associated with the supernova remnant Kes 17 was also included (Wu et al. 2011). The recommended spectral model for each source as in the 2FGL catalog was used, while we modeled PSR B1259-63 and Kes 17 with a power law (PL)

$$
\frac{d N}{d E}=N_{0}\left(\frac{E}{E_{0}}\right)^{-\Gamma} .
$$

The spectral values of PSR B1259-63 and other sources within $5^{\circ}$ from PSR B1259-63, as well as normalization parameter values for the Galactic and isotropic diffuse components, and

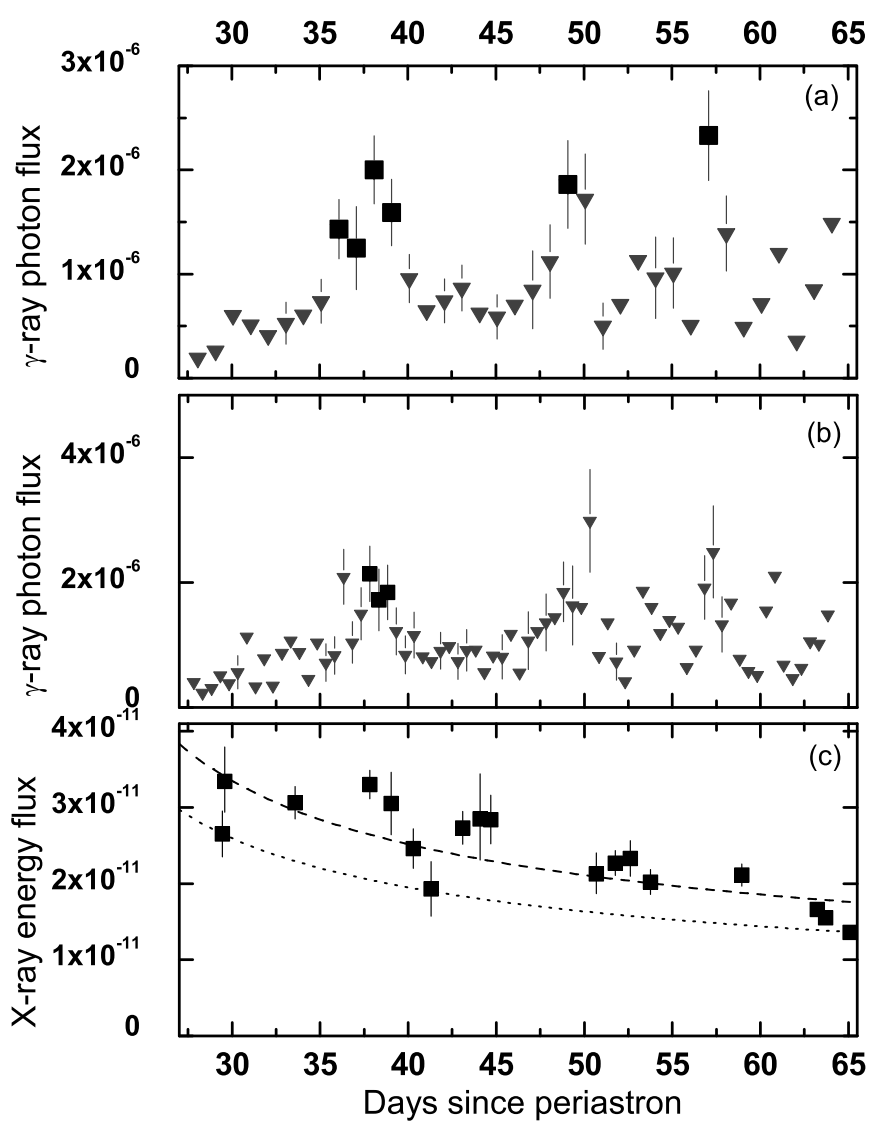

Figure 2. Light curves of PSR B1259-63/LS 2883 during the $2014 \mathrm{GeV}$ flaring period. (a) and (b): Fermi/LAT $0.1-300 \mathrm{GeV}$ light curve with daily and half-day bins, respectively. Symbols have the same meanings as in Figure 1. (c) Swift/ XRT $0.3-10 \mathrm{keV}$ light curve. The dotted and dashed lines are the same ones shown in Figure 1(e).

sources in the annulus of inner and outer radii of $5^{\circ}$ and $10^{\circ}$ from PSR B1259-63, were allowed to vary. Other parameters were fixed.

PSR B 1259-63/LS 2883 was detected with TS $=32.8$ in this analysis. The source model thus obtained was used as a template for analyses hereafter. The normalization parameter values for PSR B1259-63, the Galactic diffuse component, and sources within $4^{\circ}$ from PSR B1259-63 and the two sources marked as variable (i.e., 2FGL J1329.2-5608 and 2FGL J1330.1-7002) in the 2FGL catalog, were allowed to vary in subsequent analyses.

\subsection{Light Curve}

We derived the $0.1-300 \mathrm{GeV}$ light curve composed of five-day bins (Figure 1), assuming PL for PSR B1259-63/LS 2883. The photon indices for bins with TS $>5$ are presented as well. The light curve around the 2010 passage is shown for comparison.

As shown in Figure 1, no significant emission was found in any time intervals before and during the passage on 2014 May 4, except for the single bin with $\mathrm{TS}=9.6$ centered at $t_{\mathrm{p}}-17.5$ days. The source only became significantly detected from 2014 June 6 (i.e., $t_{\mathrm{p}}+33$ days) on (Tam et al. 2014; Wood et al. 2014), similar to the orbital phase when the last GeV flare was observed in 2011. The major flaring period continues up to around $t_{\mathrm{p}}+60$ days.

To probe shorter timescale variability during the flares, we also derived 0.1-300 GeV light curves composed of 1 day/ $12 \mathrm{hr}$ bins (Figure 2), fixing the PL index of PSR B1259-63 at 3.0 (the average photon index during the 2014 flare period). 
Table 1

0.1-300 GeV Spectral Properties During the 2014 Flaring Period

\begin{tabular}{|c|c|c|c|c|c|c|c|c|}
\hline Period & Date & Model & $\begin{array}{l}\text { Photon Flux } \\
\left(\mathrm{cm}^{-2} \mathrm{~s}^{-1}\right)\end{array}$ & $\begin{array}{c}\text { Energy Flux } \\
\left(\mathrm{erg} \mathrm{cm}^{-2} \mathrm{~s}^{-1}\right)\end{array}$ & Photon Index & $\begin{array}{c}\text { Cutoff Energy } \\
(\mathrm{MeV})\end{array}$ & $\mathrm{TS}$ & $\begin{array}{c}\mathrm{TS}_{\text {cutoff }} \\
\left(\mathrm{TS}_{\mathrm{PLE}}-\mathrm{TS}_{\mathrm{PL}}\right)\end{array}$ \\
\hline \multirow[t]{2}{*}{$\mathrm{f} 1$} & June 6-June 16 & PL & $(1.16 \pm 0.09) \times 10^{-6}$ & $(3.59 \pm 0.29) \times 10^{-10}$ & $3.07 \pm 0.10$ & & 318.0 & \\
\hline & & PLE & $(1.17 \pm 0.07) \times 10^{-6}$ & $(3.50 \pm 0.27) \times 10^{-10}$ & $2.12 \pm 0.41$ & $331 \pm 164$ & 378.5 & 60.5 \\
\hline \multirow[t]{2}{*}{$\mathrm{f} 2$} & June 17 - June 25 & PL & $(7.28 \pm 1.32) \times 10^{-7}$ & $(2.61 \pm 0.42) \times 10^{-10}$ & $2.63 \pm 0.13$ & & 84.0 & \\
\hline & & PLE & $(6.91 \pm 0.14) \times 10^{-7}$ & $(2.76 \pm 0.45) \times 10^{-10}$ & $2.26 \pm 0.31$ & $1899 \pm 1810$ & 83.4 & a \\
\hline \multirow[t]{2}{*}{$\mathrm{f} 3$} & June 26 - July 2 & PL & $(1.20 \pm 0.22) \times 10^{-6}$ & $(4.04 \pm 0.73) \times 10^{-10}$ & $2.90 \pm 0.17$ & & 88.7 & \\
\hline & & PLE & $(1.14 \pm 0.24) \times 10^{-6}$ & $(3.95 \pm 0.70) \times 10^{-10}$ & $2.37 \pm 0.44$ & $1066 \pm 914$ & 87.4 & a \\
\hline \multirow[t]{2}{*}{ Flare } & June 2 - July 2 & PL & $(9.16 \pm 0.76) \times 10^{-7}$ & $(3.10 \pm 0.24) \times 10^{-10}$ & $2.90 \pm 0.07$ & & 459.1 & \\
\hline & & PLE & $(9.06 \pm 0.77) \times 10^{-7}$ & $(2.94 \pm 0.22) \times 10^{-10}$ & $2.30 \pm 0.25$ & $653 \pm 298$ & 479.5 & 20.4 \\
\hline
\end{tabular}

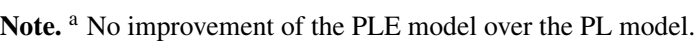

The $\gamma$-ray daily light curve is highly variable from $t_{\mathrm{p}}+30$ days to $t_{\mathrm{p}}+60$ days, clearly consisting of multiple flares. Three peaks are identified at 38 days, 49 days, and 57 days after $t_{\mathrm{p}}$, lasting for 2 to 6 days. Using $12 \mathrm{hr}$ bins, it can be seen that $\gamma$-rays from PSR B1259-63/LS 2883 undergo rapid variations down to a timescale of $12 \mathrm{hr}$. Variations at timescales down to three hours are also seen.

\subsection{Spectral Analysis During the 2014 Flaring Period}

The GeV flare formally started on 2014 June 6, but in light of the X-ray hardness revealed by the NuSTAR data (see Table 2), we regard the flaring period to be between June 2 and July 2 in this section, and the corresponding $\gamma$-ray spectrum is shown in Figure 3. Flux values of the nine energy bins were reconstructed using gtlike for each band independently, using a representative photon index of $\Gamma_{\gamma}=3$ for each bin. The PL with an exponential cutoff (PLE) model,

$$
\frac{d N}{d E}=N_{0}\left(\frac{E}{E_{0}}\right)^{-\Gamma} \exp \left(-\frac{E}{E_{\mathrm{c}}}\right),
$$

describes the $0.1-300 \mathrm{GeV}$ spectrum better than PL by $\Delta \mathrm{TS}=20.4$, i.e., $\sim 4.5 \sigma$ in significance (see Table 1 ).

We also defined three flaring episodes: (f1) June 6 to June 16; (f2) June 17 to June 25; and (f3) June 26 to July 2. We found that a PLE describes the spectrum better than a PL during $\mathrm{f} 1$, with $\Delta \mathrm{TS}=60.5$, i.e., $\sim 7.8 \sigma$ in significance. The best-fit parameters are shown in Table 1.

The 50-300 GeV bin was detected with $\mathrm{TS}=8.3$ during the flaring period, and is dominated by a $50 \mathrm{GeV}$ photon which arrived on 2014 June 10 and is located 2.4 from PSR B1259-63. We speculate that this photon is related to the spectral component in the $\mathrm{TeV}$ band at similar orbital phase (H.E.S.S. Collaboration 2013).

\subsection{Comparisons with the 2010 Periastron Passage}

Unlike the previous periastron passage, PSR B1259-63/LS 2883 was not detected by the LAT before and during the 2014 passage. This may not be too surprising given the marginal detection last time. This shows that increasing the exposure before and during the next periastron passages is very important.

The GeV flares occurred at a similar orbital phase as in early 2011, clearly demonstrating the repetitive nature of the $\mathrm{GeV}$ flares after the periastron passages in 2010 and 2014. Multipeaked structure in the light curve was first seen in 2011 (see Figure 2 of Tam et al. 2011), and is seen again this time.
Although during the first flare the flux rises at a rate slower than in 2011 January, the first peak occurs at nearly the same orbital phase $\left(t_{\mathrm{p}}+37\right.$ days and $t_{\mathrm{p}}+38$ days in 2011 and 2014, respectively).

\section{X-RAY ANALYSIS AND RESULTS}

A Swift/XRT monitoring campaign consisting of 29 observations was performed, resulting in a total exposure of $70 \mathrm{ks}$ spanning from April $20\left(t_{\mathrm{p}}-14\right.$ days $)$ to July $8\left(t_{\mathrm{p}}+65\right.$ days $)$. As the observed flux is too high during some of the observations, windowed timing (WT) mode, instead of the conventional photon counting (PC) mode, was partially used to avoid the pile-up problem. For WT data, there is an artificial turn-up in the low energy band (i.e., $<1.0 \mathrm{keV}$ ), which is a known calibration issue of this mode. ${ }^{6}$ Therefore, we excluded data below $1.0 \mathrm{keV}$ for WT data throughout the X-ray analysis, while the full band of Swift/XRT (i.e., $0.3-10 \mathrm{keV}$ ) was used for PC-mode observations.

Five NUSTAR observations were taken from 2014 April to June with a total exposure of $150 \mathrm{ks}$, which are sensitive to 3-79 keV photons.

We downloaded the Swift/XRT and NuSTAR/FPM data from the HEASARC data archive, and extracted scientific products for each observation using the tasks HEAsoft 6.14 xrtgrblc and xrtgrblcspec. Count rate-dependent regions were generated by xrtgrblc for the XRT data and by nuproducts with $30^{\prime \prime}$ radius source region and $130^{\prime \prime}$ source-free background region for the FPM data. We fit the extracted X-ray spectra with XSPEC 12.8.1.

For Swift data, because of the limited quality of an individual spectrum and the lack of soft X-rays for WT data, the absorption level of the source is hard to estimate. We therefore split the observations into two groups (i.e., pre- and post-periastron) and gave each group a common column density $\left(N_{\mathrm{H}}\right)$ for better $N_{\mathrm{H}}$ constraint. Although the interaction of PSR B1259-63/LS 2883 winds may introduce a small influence on $N_{\mathrm{H}}$, the Galactic column density toward PSR B1259-63/LS 2883 is as high as $10^{22} \mathrm{~cm}^{-2}$ (Kalberla et al. 2005), which is comparable with the best-fit $N_{\mathrm{H}} \approx 5-7 \times 10^{21} \mathrm{~cm}^{-2}$ of this source (see below), indicating that the absorption is dominated by the Galactic foreground gas and the intrinsic $N_{\mathrm{H}}$ variation of the system is negligible. All the spectra are well fit with an absorbed PL with $\chi_{v}^{2}$ values of 0.80 (dof $=34$; pre-periastron $)$ and 0.98 (dof $=674$; post-periastron). The $N_{\mathrm{H}}$ value before/after the passage is

\footnotetext{
6 http://www.swift.ac.uk/analysis/xrt/digest_cal.php
} 

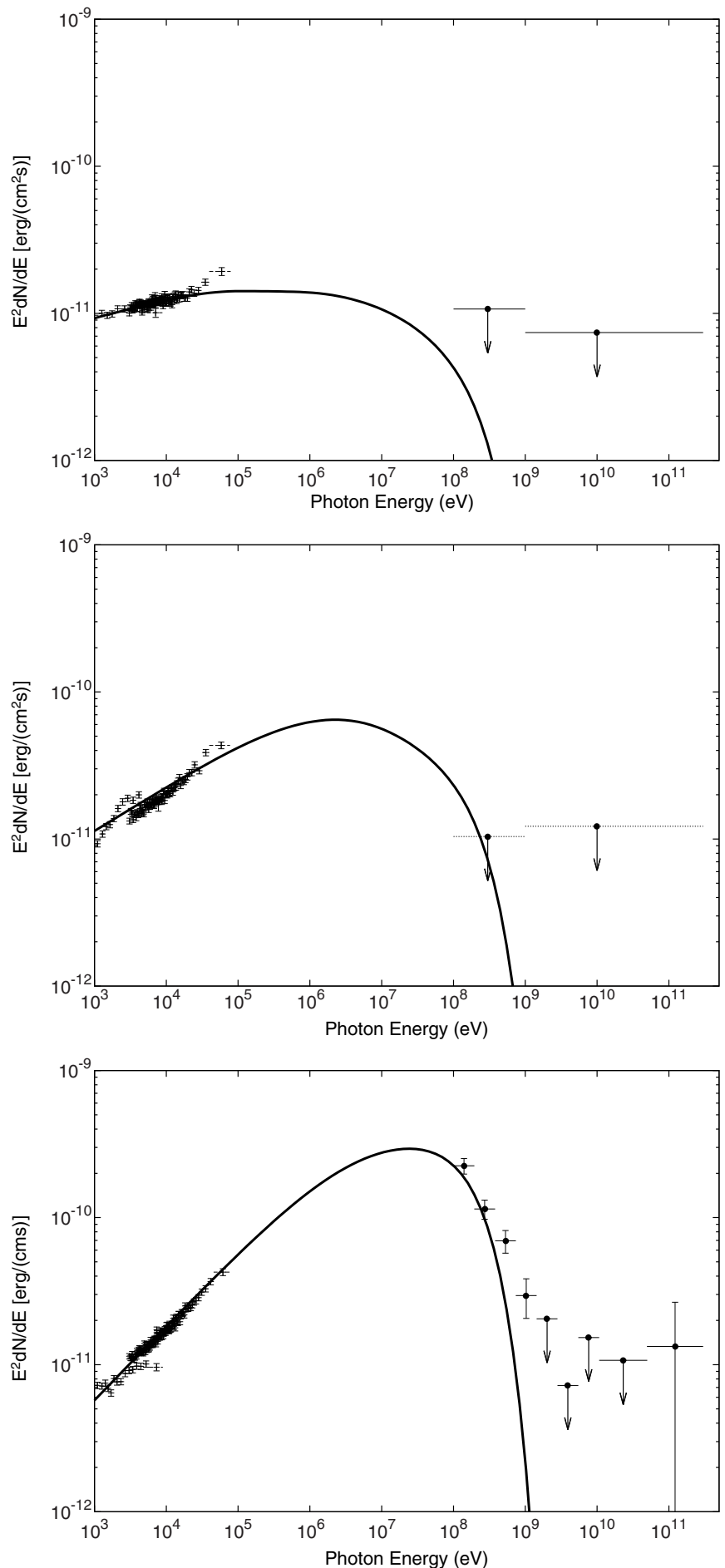

Figure 3. Spectral energy distribution from X-rays to $\gamma$-rays during phases I (periastron passage: April 20 to May 14; upper panel), II (X-ray peak: May 15 to June 1; middle panel), and III (flaring: June 2 to July 2; lower panel). Some of the X-ray error bars are smaller than the symbols. Gamma-ray data without error bars represent $95 \%$ confidence-level upper limits assuming $\Gamma=2$ for phases I and II, and $\Gamma=3$ for phase III. The solid lines show our calculated spectra of the synchrotron radiation from the shocked PW.

$6.9_{+0.09}^{-0.08} \times 10^{21} \mathrm{~cm}^{-2}$ and $5.4_{+0.05}^{-0.04} \times 10^{21} \mathrm{~cm}^{-2}$, respectively. The photon index keeps changing throughout the periastron passage, reaching $2.06_{-0.17}^{+0.18}$ on April 20, gradually dropping to $1.22_{-0.21}^{+0.21}$ on June 24 , and staying around at 1.5 afterward. Indeed, fitting the photon indices with a linear function gives
Table 2 Joint XRT/FPM 0.3-79 keV Spectral Properties

\begin{tabular}{lccc}
\hline \hline Date & $\begin{array}{c}N_{\mathrm{H}} \\
\left(\mathrm{cm}^{-2} \mathrm{~s}^{-1}\right)\end{array}$ & $\Gamma$ & $\begin{array}{c}\text { Energy Flux } \\
\left(\mathrm{erg} \mathrm{cm}^{-2} \mathrm{~s}^{-1}\right)\end{array}$ \\
\hline Apr 20 & $0.90_{-0.10}^{+0.13}$ & $1.88 \pm 0.02$ & $(8.45 \pm 0.09) \times 10^{-11}$ \\
May 4 & $0.69_{-0.06}^{+0.07}$ & $1.94 \pm 0.02$ & $(4.51 \pm 0.06) \times 10^{-11}$ \\
May 28 & $0.52_{-0.08}^{+0.09}$ & $1.66 \pm 0.02$ & $(1.02 \pm 0.01) \times 10^{-10}$ \\
Jun 2 & $0.67_{-0.15}^{+0.17}$ & $1.57 \pm 0.02$ & $(9.14 \pm 0.12) \times 10^{-11}$ \\
Jun 14 & $0.77_{-0.21}^{+0.25}$ & $1.57 \pm 0.02$ & $(8.42 \pm 0.11) \times 10^{-11}$ \\
\hline
\end{tabular}

$\Gamma_{\mathrm{XRT}}=(1.89 \pm 0.04)-(0.006 \pm 0.001)\left[t(d)-t_{\mathrm{p}}\right](\mathrm{see}$ Figure 1). The decreasing trend is confirmed with the $F$-test probability of $1.6 \times 10^{-6}$. This hardening of X-ray emission is further established by $N u S T A R$ observations. Previous X-ray observations showed similar X-ray spectral hardening through periastron passages (e.g., Chernyakova et al. 2009, 2014), and this behavior now extends to $79 \mathrm{keV}$ thanks to NuSTAR observations.

The X-ray flux variation is compatible with the previously observed double-hump structure (Chernyakova et al. 2009). Although no huge flare is detected, there is seemingly shortterm variability along the PL-like declining trend from 2014 June 2 to July 8 (panel (e) of Figure 1). X-ray variability of short timescales, i.e., down to $\sim 1 \mathrm{ks}$, was previously reported in two epochs of pre-periastron observations $\left(t_{\mathrm{p}}-19\right.$ days and $t_{\mathrm{p}}-11$ days), attributed to the clumpy structure of the stellar wind (Chernyakova et al. 2009). With data taken from the GeV flaring period June 2 to July 8 , the best-fit decay index of the trend is $-0.47 \pm 0.01$ with a $\chi_{v}^{2}=3.3(\mathrm{dof}=16)$. As the model matches well with the declining trend, we suggest that the large $\chi^{2}$ value is indeed caused by short-term variability. If so, the significance of short-term variability is more than $5 \sigma$. We speculate that the variability is mainly caused by several small-scale flaring events. We therefore construct a baseline light curve, $F \propto\left(t-t_{0}\right)^{-0.47}$, where $t_{0}$ is the observed highest peak of the light curve (see panel (e) of Figure 1; the X-ray peak at $t_{0}$ has the highest flux ever detected from PSR B1259-63/LS 2883, first noted in Tam $\&$ Kong 2014), based on the three low points of the phase (that occurred on June 2, June 14, and July 8) to investigate the amplitude of the flares. By defining a flare coefficient, $\kappa_{\mathrm{X}}$, as the ratio between the observed flux (absorption corrected) and the baseline model flux (i.e., $\kappa_{\mathrm{X}}=F_{\text {observed }} / F_{\text {baseline}}$ ), we show that the nominal flare occupies a considerable fraction of the total flux, equivalent to $10 \%-60 \%$ of the baseline flux. The flare coefficient during the $\mathrm{GeV}$ flaring period is about $\kappa_{\mathrm{X}}=1.1$ to $\kappa_{\mathrm{X}}=1.6$.

We fit the NuSTAR spectra simultaneously with the corresponding Swift/XRT data and all the combined spectra can be described by an absorbed PL (overall $\chi_{v}^{2}=0.98$ with dof $=3671$; the best-fit photon indices and fluxes are shown in Table 2). In Figure 3, we plotted three spectra using contemporaneous XRT+FPM spectra (i.e., phase I: April 20 to May 14; phase II: May 15 to June 1; and phase III: June 2 to July 2), representing the periastron passage, X-ray peak, and flaring phases, respectively.

\section{DISCUSSION}

Kong et al. (2012) proposed that the GeV flare is due to Doppler boosting of the shocked PW. In this model, the line of sight cuts through the shocked PW cone during the phase 
of the $\mathrm{GeV}$ flares, boosting the emissions through relativistic effects. However, because the Doppler boosting not only affects the $\mathrm{GeV}$ band but also the X-ray band, the peak of the GeV light curve is in phase with the post-periastron X-ray peak, which is incompatible with the observed $\mathrm{GeV} / \mathrm{X}$-ray light curves (see Figure 1). The IC scattering process of the PW has been discussed before (van Soelen et al. 2012; Khangulyan et al. 2012; Dubus \& Cerutti 2013; Mochol \& Kirk 2013), while different seed photons are assumed in different models. van Soelen et al. (2012) calculated the IC scattering process between the PW and infrared photons from the Be disk. In the $\gamma$-ray light curve, the disk contribution is at the maximum at the periastron passage because the disk is the brightest near the stellar surface. Dubus \& Cerutti (2013) discussed the IC scattering of the PW off the X-rays from the shocked PW. However, this model does not explain the observed temporal delay between the X-ray peak and GeV flare. Khangulyan et al. (2012) proposed that the $\mathrm{GeV}$ flare occurs as a consequence of the rapid changing of shock when the pulsar exits the stellar disk. The opening of the shock cone enables the cold-relativistic PW to travel further along the line of sight, and to cool through the IC scattering off the optical photons from the disk. This model can explain the delay between the GeV flare and the X-ray peak. In this model, a large energy density of the seed photons reaching about 10 times greater than the stellar radiation density is required (see Dubus \& Cerutti 2013). However, observations through previous periastron passages do not seem to suggest such a large heating effect (Negueruela et al. 2011) and dedicated optical and IR observations during the Fermi flare are required to investigate the heating of the disk matter by the PW. A hydrodynamic simulation (Takata et al. 2012) suggests that the disk matter is deformed by the pulsar and creates a cavity structure around the pulsar. The cavity structure may allow the head-on collision between the PW and the IR photons from the heated matter, increasing the efficiency of $\gamma$-ray production.

As shown in Table 2 and Figure 1, new X-ray observations indicate the hardening of spectra up to $79 \mathrm{keV}$ over time, and the linear extrapolation of the X-ray spectrum to the high-energy band roughly connects to the Fermi data during phase III (see Figure 3), suggesting that $\mathrm{X}$-ray and $\mathrm{GeV}$ emissions are related to each other, and $\mathrm{GeV}$ flare emission likely originates from the synchrotron radiation processes. Chernyakova et al. (2014) proposed a synchrotron radiation scenario in which the fly-by of the disk material disturbs the magnetic field of the un-shocked PW and produces the pitch angle of the PW particles, which can be cooled down through the synchrotron radiation.

In Figure 3, we compare our calculated spectra of the synchrotron radiation from the shocked $\mathrm{PW}$ with the observed spectra before and during the $\mathrm{GeV}$ flares. We used the shock structure obtained by a three-dimensional smoothed particle hydrodynamics simulation for the Be-disk base density of $10^{-9} \mathrm{~g} \mathrm{~cm}^{-3}$ (A. T. Okazaki et al., in preparation). This simulation has been carried out using the same model as in Takata et al. (2012), but with updated stellar and wind parameters (Negueruela et al. 2011; Moldón et al. 2011). We assumed that the PW is accelerated at the shock and the maximum Lorentz factor is determined by balancing the accretion timescale $\left(\gamma m_{e} c / e B\right)$ with the synchrotron loss (or dynamical) timescale. The typical Lorentz factor in this work is of the order $\gamma_{\max } \sim 10^{8}$ and makes a spectral break of the synchrotron radiation at $10-100 \mathrm{MeV}$. We chose the power index, $p$, of the shocked particles to explain the $\mathrm{X}$-ray spectral index, and we assumed $p=2.7$ for phase I, $p=2.4$ for phase II, and $p=2.0$ for phase III. In calculating the synchrotron emission with the result of hydrodynamic simulation, we assumed that the total PW pressure at each simulation grid is associated with $20 \%$ of the magnetic pressure and $80 \%$ of the particle pressure. The typical magnetic field at the shock region in this work is $B \sim 0.05-1$ gauss. We found that if the magnetic field at the shock is $B \ll 0.1$ gauss, the calculated flux is well below the observed flux. We refer to Takata et al. (2012) for details of the calculations. This model interprets the preand post-periastron $\mathrm{X}$-ray peaks as due to significant increase of the conversion efficiency from pulsar spin-down power to the shock-accelerated particle energy at orbital phases when the pulsar crosses the disk.

We acknowledge the use of data and software facilities from the FSSC, managed by the HEASARC at the Goddard Space Flight Center. P.H.T. and K.L.L. are supported by the Ministry of Science and Technology (MOST) of the Republic of China (Taiwan) through grant 101-2112-M-007-022-MY3. A.K.H.K. is supported by MOST through grants 100-2628M-007-002-MY3, 100-2923-M-007-001-MY3, and 103-2628M-007-003-MY3. J.T. is supported by a 2014 GRF grant of Hong Kong Government under HKU 17300814P. C.Y.H. is supported by the National Research Foundation of Korea through grant 2014R1A1A2058590.

\section{REFERENCES}

Abdo, A. A., Ackermann, M., Ajello, M., et al. (Fermi/LAT Collaboration) 2011, ApJL, 736, L11

Aharonian, F. A., Akhperjanian, A. G., Anton, G., et al. (H.E.S.S. Collaboration) 2009, A\&A, 507, 389

Aharonian, F. A., Akhperjanian, A. G., Aye, K.-M., et al. (H.E.S.S. Collaboration) 2005, A\&A, 442, 1

Atwood, W. B., Abdo, A. A., Ackermann, M., et al. (Fermi/LAT Collaboration) 2009, ApJ, 697, 1071

Bogovalov, S. V., Khangulyan, D. V., Koldoba, A. V., Ustyugova, G. V., \& Aharonian, F. A. 2008, MNRAS, 387, 63

Chernyakova, M., Abdo, A. A., Neronov, A., et al. 2014, MNRAS, 439, 432

Chernyakova, M., Neronov, A., Aharonian, F., Uchiyama, Y., \& Takahashi, T. 2009, MNRAS, 397, 2123

Chernyakova, M., Neronov, A., Lutovinov, A., Rodriguez, J., \& Revnivtsev, M 2006, MNRAS, 367, 1201

Dubus, G. 2006, A\&A, 451, 9

Dubus, G., \& Cerutti, B. 2013, A\&A, 557, A127

Dubus, G., Cerutti, B., \& Henri, G. 2010, A\&A, 516, 18

H.E.S.S. Collaboration 2013, A\&A, 551, A94

Johnston, S., Ball, L., Wang, N., \& Manchester, R. N. 2005, MNRAS, 358, 1069

Johnston, S., Manchester, R. N., Lyne, A. G., Nicastro, L., \& Spyromilio, J. 1994, MNRAS, 268, 430

Kalberla, P. M. W., Burton, W. B., Hartmann, D., et al. 2005, A\&A, 440, 775

Khangulyan, D., Aharonian, F. A., Bogovalov, S. V., \& Ribó, M. 2011, ApJ, 742,98

Khangulyan, D., Aharonian, F. A., Bogovalov, S. V., \& Ribó, M. 2012, ApJL, 752, L17

Kirk, J. G., Ball, L., \& Skjæraasen, O. 1999, APh, 10, 31

Kong, A. K. H., Huang, R. H. H., Tam, P. H. T., \& Hui, C. Y. 2011 a, ATel, 3111

Kong, S. W., Cheng, K. S., \& Huang, Y. F. 2012, ApJ, 753, 127

Kong, S. W., Yu, Y. W., Huang, Y. F., \& Cheng, K. S. 2011b, MNRAS, 416, 1067

Mochol, I., \& Kirk, J. G. 2013, ApJ, 776, 40

Moldón, J., Johnston, S., Ribó, M., Paredes, J. M., \& Deller, A. T. 2011, ApJL, 732, L10

Negueruela, I., Ribó, M., Lorenzo, J., khangulyan, D., \& Aharonian, F. A. 2011, ApJL, 732, L11

Nolan, P. L., Abdo, A. A., Ackermann, M., et al. 2012, ApJS, 199, 31

Pavlov, G. G., Chang, C., \& Kargaltsev, O. 2011, ApJ, 730, 2

Shannon, R. M., Johnston, S., \& Manchester, R. N. 2014, MNRAS, 437, 3255

Takata, J., Okazaki, A. T., Nagataki, S., et al. 2012, ApJ, 750, 70 
Takata, J., \& Taam, R. E. 2009, ApJ, 702, 100

Tam, P. H. T., Huang, R. H. H., Takata, J., et al. 2011, ApJL, 736, L10

Tam, P. H. T., \& Kong, A. K. H. 2014, ATel, 6198, 1

Tam, P. H. T., Kong, A. K. H., \& Leung, G. C. K. 2014, ATel, 6216, 1

Tavani, M., \& Arons, J. 1997, ApJ, 477, 439 van Soelen, B., Meintjes, P. J., Odendaal, A., \& Townsend, L. J. 2012, MNRAS, 426,3135

Wex, N., Johnston, S., Manchester, R. N., et al. 1998, MNRAS, 298, 997 Wood, K. S., Caliandro, G. A., Cheung, C. C., Li, J., Torres, D. F., \& (Fermi LAT Collaboration) 2014, ATel, 6225, 1

Wu, J. H. K., Wu, E. M. H., Hui, C. Y., et al. 2011, ApJL, 740, L12 